Case Report

\title{
Clear Cell Odontogenic Carcinoma of the Mandible Harboring EWSR1 Rearrangement: Report of a Massive Jaw Tumor and Review of Diagnostic Considerations
}

\author{
Adepitan A. Owosho ${ }^{1},{ }^{1}$ Chukwubuzor Okwuosa, ${ }^{2}$ Donald I. Obi, ${ }^{2}$ Robinson O. Okiti, ${ }^{3}$ \\ and Kurt F. Summersgill ${ }^{4}$ \\ ${ }^{1}$ Missouri School of Dentistry and Oral Health, A.T. Still University, Kirksville, Missouri, USA \\ ${ }^{2}$ University of Nigeria Teaching Hospital, Nsukka, Enugu, Nigeria \\ ${ }^{3}$ University College Hospital, Ibadan, Oyo, Nigeria \\ ${ }^{4}$ Department of Diagnostic Sciences, School of Dental Medicine, University of Pittsburgh, Pittsburgh, Pennsylvania, USA
}

Correspondence should be addressed to Adepitan A. Owosho; adepitanowosho@atsu.edu

Received 21 January 2021; Revised 26 February 2021; Accepted 3 March 2021; Published 9 March 2021

Academic Editor: Eugenio Maiorano

Copyright (c) 2021 Adepitan A. Owosho et al. This is an open access article distributed under the Creative Commons Attribution License, which permits unrestricted use, distribution, and reproduction in any medium, provided the original work is properly cited.

\begin{abstract}
Clear cell odontogenic carcinoma (CCOC) is a low-grade malignant neoplasm that affects the jaws. We report an $18 \mathrm{~cm}$ massive case of mandibular CCOC in a 43-year-old female. The tumor was composed of nests and cords of round to polygonal monomorphic clear cells separated by prominent stromal hyalinization. Immunohistochemically, the tumor cells showed focal cytokeratin 5/6 positivity and intracytoplasmic PAS-positive granules and were negative for S100 and after diastase treatment (PAS-D). Molecularly, this case was positive for EWSR1 rearrangement by FISH. The following should be included in the histopathological differential diagnosis: hyalinizing clear cell carcinoma of the salivary gland, clear cell variant of central mucoepidermoid carcinoma, clear cell variant of calcifying epithelial odontogenic tumor, and metastatic renal cell carcinoma. CCOC is a rare entity, with only 79 cases reported in the mandible. This case highlights the propensity for CCOC to exhibit invasiveness, destructive nature, and facial disfigurement if left untreated.
\end{abstract}

\section{Introduction}

Clear cell odontogenic carcinoma (CCOC) is a rare malignant odontogenic tumor with less 120 cases reported in the jaws since it was first described by Hansen et al. in 1985 [1]. CCOC was previously known as clear cell odontogenic tumor or clear cell ameloblastoma, when it was classified as a benign neoplasm $[1,2]$. However, due to its aggressive clinical characteristics such as invasive growth, regional lymph node involvement, local recurrence, and distant metastasis, the WHO reclassified it as a malignant tumor of odontogenic origin in 2005. CCOC has a preference for females in the 5th decade of life and for the mandible, with $73.8 \%$ of reported cases occurring in the mandible [3].
Morphologically, CCOC presents as an infiltrative tumor composed of nests, sheets, or cords of monomorphic round to polygonal clear epithelial cells separated by variable hyalinized stroma [4]. Immunophenotypically, they are positive for cytokeratins, p63, and PAS, are diastase-sensitive (demonstrating intracytoplasmic glycogen), and are negative for mucicarmine and myoepithelial markers such as S100, SMA, and calponin $[4,5]$. Molecularly, CCOC has been shown to demonstrate Ewing sarcoma region 1 (EWSR1) rearrangement [5]. In this article, we report a massive case of CCOC of the mandible in a Nigerian patient, performing limited immunohistochemical stains (CK5/6 and S100), special stains (PAS and, PAS-D), and FISH molecular analysis for EWSR1 rearrangement. 


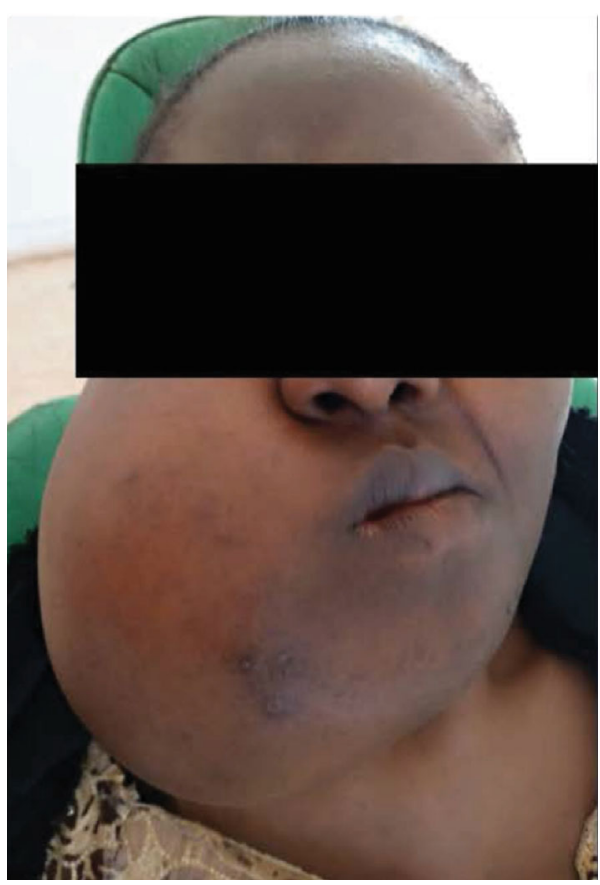

(a)

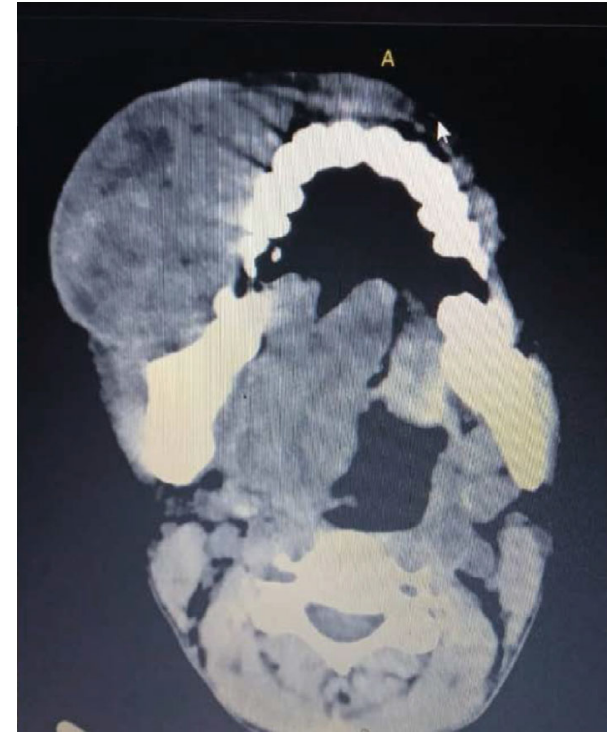

(b)

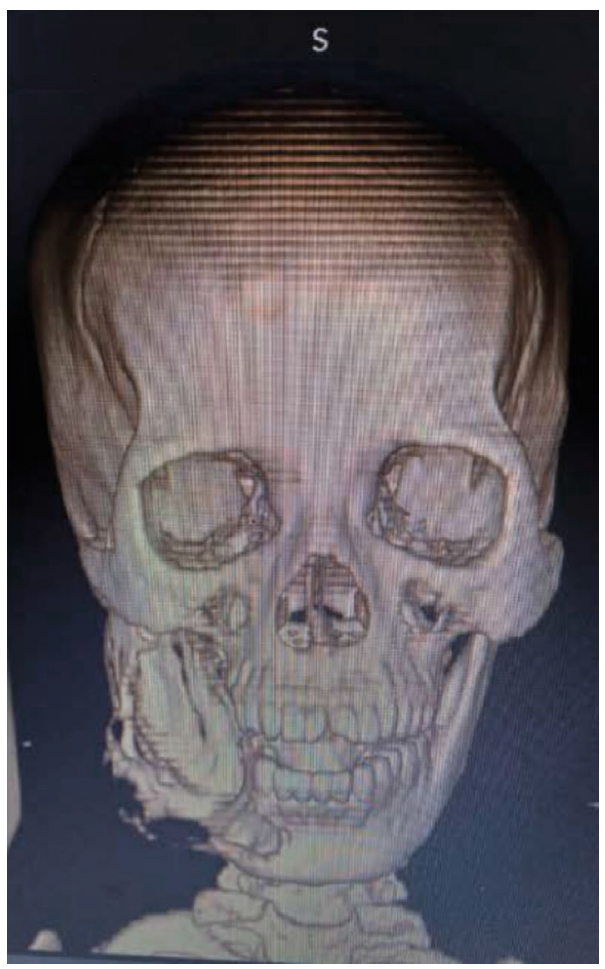

(c)

FIGURE 1: Clinical image of a 43-year-old female patient diagnosed with a EWSR1-rearranged clear cell odontogenic carcinoma of the mandible (a), CT scan axial view (b), and 3D-refomatted CT scan showing an osteolytic destructive mass in the mandible with thinning and perforation of the cortical border of the mandible (c).

\section{Case Report}

A 43-year-old female patient presented in September 2019 with a 4-year history of a mandibular mass of $18 \mathrm{~cm}$ in diameter associated with buccolingual expansion of the mandible
(Figure 1(a)) and regional lymph node involvement. Incisional biopsy had been performed 2 years prior, and an initial diagnosis of ameloblastoma was rendered. The mass was reported to have progressively increased in size for the past 2 years prior to presentation. Imaging revealed an osteolytic 


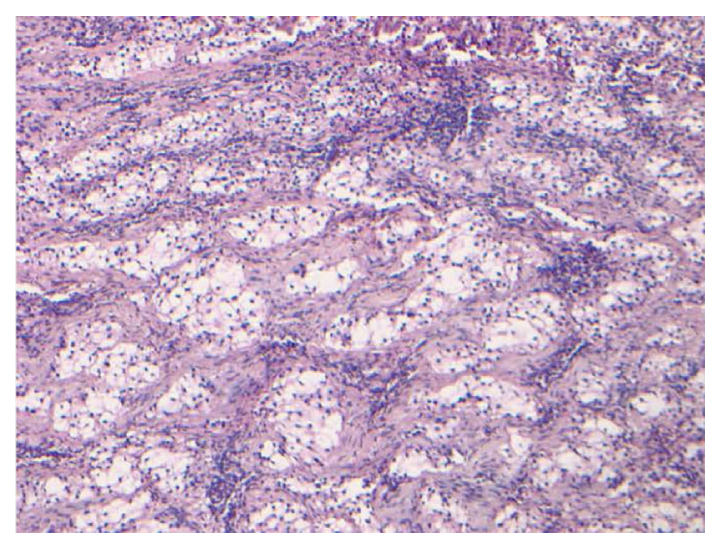

(a)

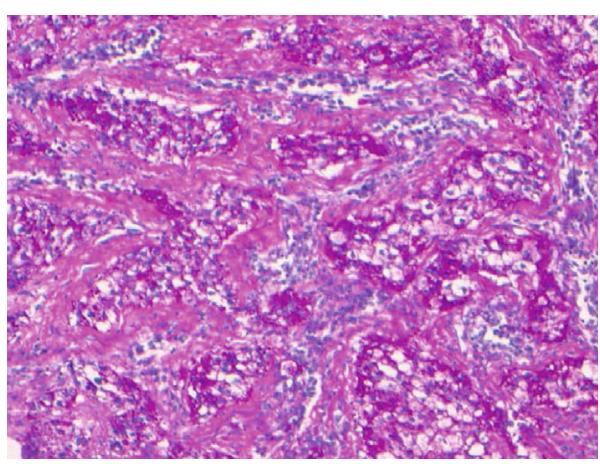

(c)

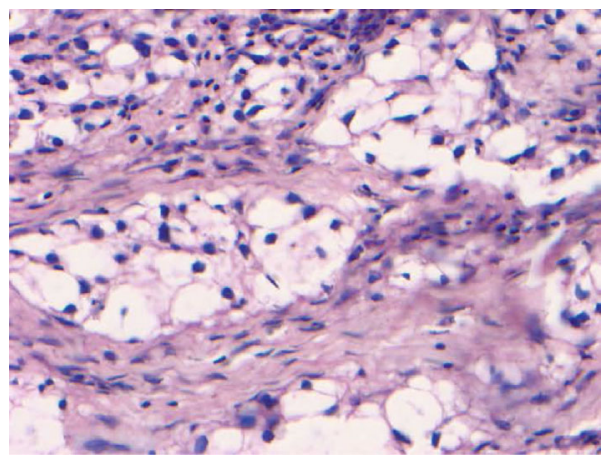

(b)

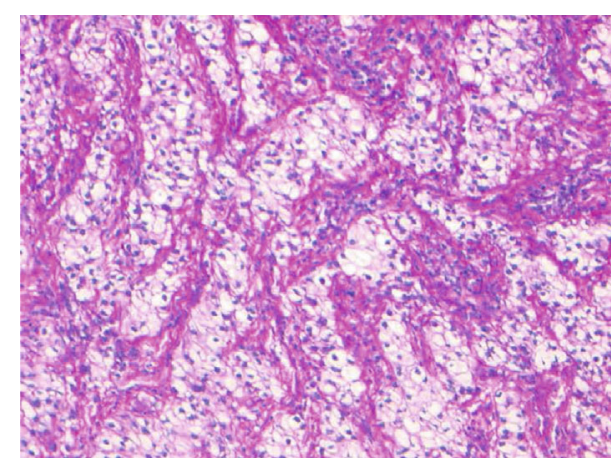

(d)

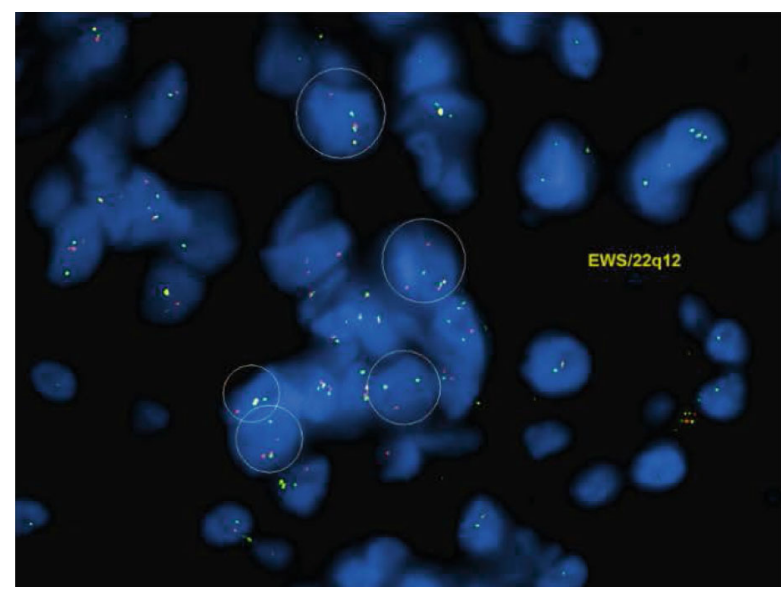

(e)

Figure 2: Histopathology of clear cell odontogenic carcinoma of the mandible in a 43-year-old female patient. (a) Morphology shows nests and cords of round to polygonal monomorphic clear epithelial cells with prominent stromal hyalinization containing lymphocytes (H\&E 100x). (b) Higher magnification (H\&E 400x). (c) Clear epithelial cells positive for PAS demonstrating intracytoplasmic granules (PAS 200x). (d) Clear epithelial cells are negative after PAS-D (200x). (e) Split of the green signal from the red signal by break-apart FISH demonstrates that the tumor harbors EWSR1 rearrangement. In contrast, the uninvolved EWSR1 allele shows a yellow signal (resulting from the fused red and green probes).

destructive mass in the mandible with thinning and perforation of the cortical border of the mandible (Figures 1(b) and 1(c)). A rebiopsy was performed.

Morphologic features of the tumor were of nests and cords of round to polygonal monomorphic clear epithelial cells with prominent stromal hyalinization (Figures 2(a) and 2(b)). The tumor was poorly circumscribed and infiltrative with the presence of lymphocytes in the stroma.
The tumor was positive for PAS but negative for PAS-D (demonstrating the presence of intracytoplasmic glycogen) (Figures 2(c) and 2(d)), showed focal expression for CK5/6, and was negative for $\mathrm{S} 100$ protein. FISH for EWSR1 rearrangement was positive with $65 \%$ of translocated cells in 60 cells examined (Figure 2(e)). The cut-off for a positive EWSR1 rearrangement is $12.5 \%$ of cells translocated. 


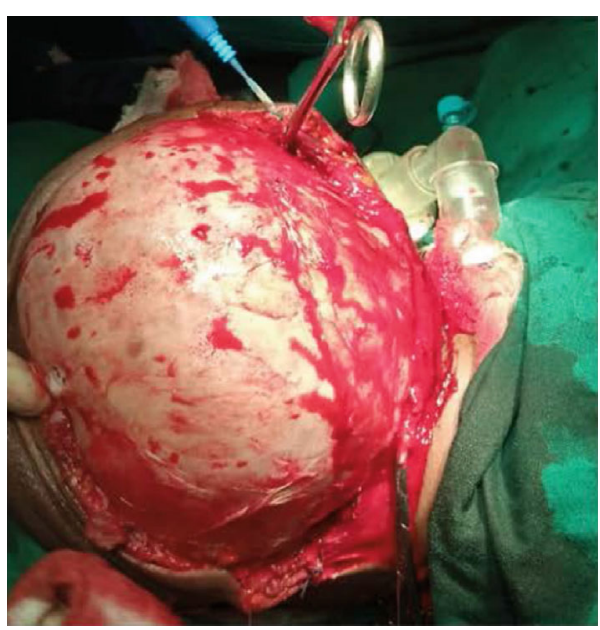

(a)

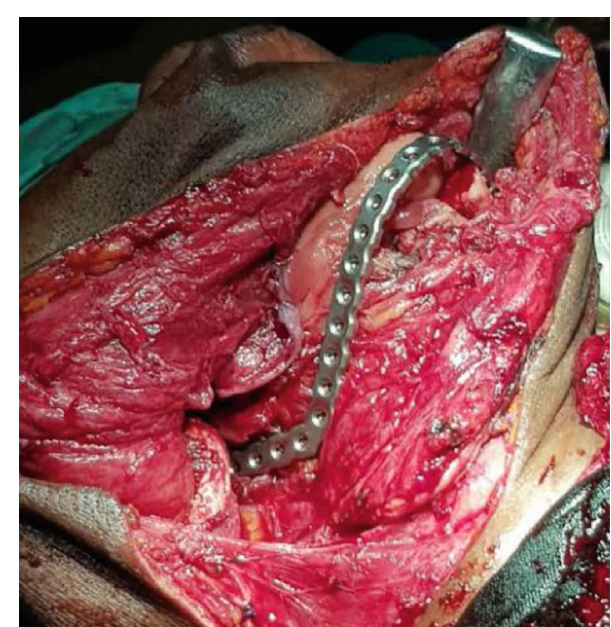

(b)

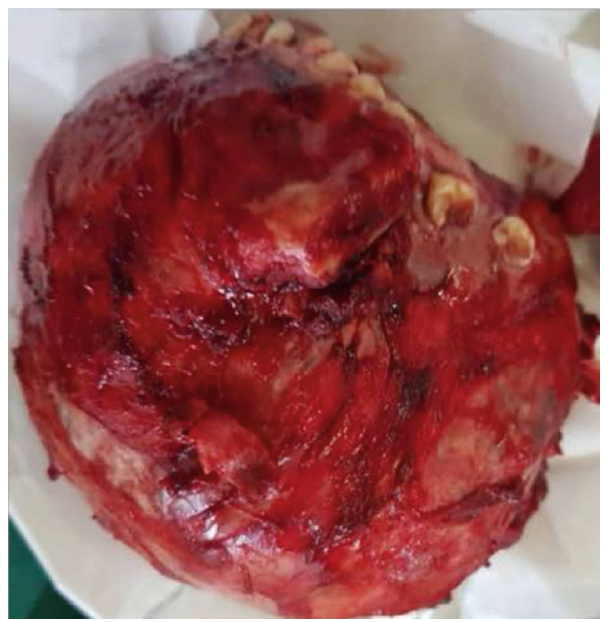

(c)

FIGURE 3: (a) Surgical exposure and resection of the EWSR1-rearranged clear cell odontogenic carcinoma of the mandible. (b) Reconstruction of the surgical defect with a mandibular reconstruction plate. (c) Massive resected EWSR1-rearranged clear cell odontogenic carcinoma of the mandible.

Two months after presentation, a subtotal mandibulectomy with neck dissection was performed for the resection of the CCOC (Figures 3(a)-3(c)). The patient is alive without disease and no recurrence at the last follow-up 15 months posttherapy.

\section{Discussion}

Clear cell odontogenic carcinoma (CCOC) is a rare malignant odontogenic neoplasm characterized by EWSR1 gene rearrangement. Less than 120 cases have been reported in the literature. A recent review of the literature of 107 patients diagnosed with CCOC showed a predilection for females, the 5th decade of life, and the mandible [3]. In the same review, $13.6 \%(12 / 88)$ of CCOC patients manifested regional nodal involvement, and $11.2 \%(12 / 107)$ of CCOC patients died of disease [3]. In this report, we present a case of CCOC in a 43 -year-old female with progressive growing mass, $18 \mathrm{~cm}$ in size in its largest diameter, located in the mandible associated with regional lymph node involvement and alive without disease and no recurrence 15 months posttherapy.

EWSR1 rearrangement in CCOC was first described by Bilodeau et al.; the fusion partner identified was the activating transcription factor 1 gene $(A T F 1)$ on chromosome $12 \mathrm{q} 13$ [5]. An alternative fusion partner, $C R E B 1$, on chromosome 2q34 has been described [6]. ATF1 and CREB1 both belong to the cAMP response element-binding protein (CREB) family of transcription factors. ATF1 remains the most common fusion partner to EWSR 1 identified in CCOC to date. Most cases of CCOC reported in the literature were described before EWSR1 rearrangement was identified in the tumor. EWSR1 gene rearrangement has been reported in numerous tumors, such as Ewing sarcoma, extraskeletal myxoid chondrosarcoma, clear cell sarcoma, desmoplastic small round cell tumor, myxoid liposarcoma, angiomatoid fibrous histiocytoma, primary pulmonary myxoid sarcoma, myoepithelial tumors of soft tissue, skin, and salivary glands, and hyalinizing clear cell carcinoma. EWSR1-ATF1 translocation has been reported in other tumors, such as 
angiomatoid fibrous histiocytoma, hyalinizing clear cell carcinoma, and clear cell sarcoma [7-9]. Additionally, EWSR1CREB1 translocation has been reported in myxoid mesenchymal tumors, angiomatoid fibrous histiocytoma, primary pulmonary myxoid sarcoma, and gastrointestinal-clear cell sarcoma [10-13].

The differential diagnosis of CCOC includes hyalinizing clear cell carcinoma (HCCC) of the salivary gland, clear cell variant of central mucoepidermoid carcinoma (MEC), clear cell variant of calcifying epithelial odontogenic tumor (CEOT), and metastatic renal cell carcinoma. HCCC of the salivary gland is a rare low-grade malignant neoplasm that typically affects the minor salivary glands [14]. It has been argued that CCOC and HCCC are essentially the same analogous tumors with different locations $[4,5]$. CCOC occurs centrally in the bone while HCCC occurs peripherally in the submucosa. CCOC and HCCC share similar morphologic, immunophenotypic, and molecular features [5].

MEC is the most common malignant salivary gland tumor that needs to be differentiated from CCOC, despite the presence of intercellular bridging of true epidermoid areas. MEC is composed of epidermoid, mucinous, and intermediate cells in variable proportion depending on its grade, forming solid and cystic patterns. MEC has been described in the jaw bone [15]. Clear cells have been described in MEC, and when predominant, they are called the clear cell variant of MEC. Immunohistochemical stains are not helpful in differentiating MEC from CCOC. However, PAS-D shows positive intracytoplasmic globules in MEC, which are negative in CCOC $[4,16]$. MEC is negative for EWSR1 rearrangement but may be positive for CRTC1/CRTC3-MAML2 fusion [17].

CEOT is a rare invasive epithelial odontogenic tumor also known as Pindborg tumor. CEOT is composed of sheets, nests, and/or cords of polygonal eosinophilic epithelial cells with a prominent stroma [18]. These epithelial cells may present with clear cytoplasm, making it challenging to differentiate it from CCOC. However, cellular pleomorphism and the presence of amyloid and Liesegang calcified rings in the stroma that define CEOT are absent in CCOC. The eosinophilic amyloid material can be highlighted with Congo red stain, showing the classic apple-green birefringence with polarized light.

Metastasis of renal cell carcinoma to jaw should also be considered in the differential diagnosis of CCOC. These patients may have a history of a renal mass, or the metastasis to the oral cavity may be the first indication of a malignancy [19]. The metastatic renal cell carcinoma will be positive for PAX8, CD10, and CAIX in contrast to CCOC and negative for EWSR1 rearrangement.

The case described here originated from the jaw, negative for PAS-D, and positive for PAS and EWSR1 rearrangement, ruling out the aforementioned differential diagnoses. In conclusion, we report a rare form of jaw neoplasm "clear cell odontogenic carcinoma" with a propensity for invasiveness, destructive nature, and facial disfigurement if left untreated in a Nigerian patient harboring EWSR1 rearrangement.

\section{Data Availability}

No data were used to support this study.

\section{Disclosure}

The funding source has no role in conceiving and performing the study.

\section{Conflicts of Interest}

The authors declare that they have no conflicts of interest.

\section{Authors' Contributions}

Adepitan A. Owosho and Chukwubuzor Okwuosa are coequal first authors.

\section{References}

[1] L. S. Hansen, L. R. Eversole, T. L. Green, and N. B. Powell, "Clear cell odontogenic tumor-a new histologic variant with aggressive potential," Head \& Neck Surgery, vol. 8, no. 2, pp. 115-123, 1985.

[2] C. A. Waldron, I. A. Small, and H. Silverman, "Clear cell ameloblastoma-an odontogenic carcinoma," Journal of Oral and Maxillofacial Surgery, vol. 43, no. 9, pp. 707-717, 1985.

[3] F. P. S. Guastaldi, W. C. Faquin, F. Gootkind et al., "Clear cell odontogenic carcinoma: a rare jaw tumor. A summary of 107 reported cases," International Journal of Oral and Maxillofacial Surgery, vol. 48, no. 11, pp. 1405-1410, 2019.

[4] E. A. Bilodeau, A. P. Hoschar, E. L. Barnes, J. L. Hunt, and R. R. Seethala, "Clear cell carcinoma and clear cell odontogenic carcinoma: a comparative clinicopathologic and immunohistochemical study," Head and Neck Pathology, vol. 5, no. 2, pp. 101-107, 2011.

[5] E. A. Bilodeau, I. Weinreb, C. R. Antonescu et al., "Clear cell odontogenic carcinomas show EWSR1 rearrangements," The American Journal of Surgical Pathology, vol. 37, no. 7, pp. 1001-1005, 2013.

[6] R. Vogels, D. Baumhoer, J. van Gorp et al., "Clear cell odontogenic carcinoma: occurrence of EWSR1-CREB1 as alternative fusion gene to EWSR1-ATF1," Head and Neck Pathology, vol. 13, no. 2, pp. 225-230, 2019.

[7] K. H. Hallor, F. Mertens, Y. Jin et al., "Fusion of the EWSR1 and ATF1 genes without expression of the MITF-M transcript in angiomatoid fibrous histiocytoma," Genes, Chromosomes \& Cancer, vol. 44, no. 1, pp. 97-102, 2005.

[8] C. R. Antonescu, N. Katabi, L. Zhang et al., "EWSR1-ATF1 fusion is a novel and consistent finding in hyalinizing clearcell carcinoma of salivary gland," Genes, Chromosomes \& Cancer, vol. 50, no. 7, pp. 559-570, 2011.

[9] I. Panagopoulos, F. Mertens, M. Dêbiec-Rychter et al., "Molecular genetic characterization of the EWS/ATF1 fusion gene in clear cell sarcoma of tendons and aponeuroses," International Journal of Cancer, vol. 99, no. 4, pp. 560-567, 2002.

[10] Y. C. Kao, Y. S. Sung, L. Zhang et al., "EWSR1 fusions with CREB family transcription factors define a novel myxoid mesenchymal tumor with predilection for intracranial location," The American Journal of Surgical Pathology, vol. 41, no. 4, pp. 482-490, 2017. 
[11] C. R. Antonescu, P. Dal Cin, K. Nafa et al., "EWSR1-CREB1 is the predominant gene fusion in angiomatoid fibrous histiocytoma," Genes, Chromosomes \& Cancer, vol. 46, no. 12, pp. 1051-1060, 2007.

[12] C. R. Antonescu, K. Nafa, N. H. Segal, P. Dal Cin, and M. Ladanyi, "EWS-CREB1: a recurrent variant fusion in clear cell sarcoma-association with gastrointestinal location and absence of melanocytic differentiation," Clinical Cancer Research, vol. 12, no. 18, pp. 5356-5362, 2006.

[13] K. Thway, A. G. Nicholson, K. Lawson et al., "Primary pulmonary myxoid sarcoma with EWSR1-CREB1 fusion: a new tumor entity," The American Journal of Surgical Pathology, vol. 35, no. 11, pp. 1722-1732, 2011.

[14] X. H. Yang, L. Liu, Y. Y. Shi, Y. J. Hu, Q. G. Hu, and P. Zhang, "Hyalinizing clear cell carcinoma of salivary gland origin in the head and neck: clinical and histopathological analysis," International Journal of Oral and Maxillofacial Surgery, vol. 47, no. 6, pp. 692-698, 2018.

[15] C. A. Waldron and M. L. Koh, "Central mucoepidermoid carcinoma of the jaws: report of four cases with analysis of the literature and discussion of the relationship to mucoepidermoid, sialodontogenic, and glandular odontogenic cysts," Journal of Oral and Maxillofacial Surgery, vol. 48, no. 8, pp. 871-877, 1990.

[16] A. C. Buch, P. H. Jariwala, N. K. Panicker, and M. Joshi, “The utility of periodic acid schiff with diastase and alcian blue stains on fine needle aspirates of breast and salivary gland neoplasms," Journal of Cytology, vol. 29, no. 4, pp. 221-225, 2012.

[17] G. Tonon, S. Modi, L. Wu et al., "T(11;19)(q21;p13) translocation in mucoepidermoid carcinoma creates a novel fusion product that disrupts a notch signaling pathway," Nature Genetics, vol. 33, no. 2, pp. 208-213, 2003.

[18] C. D. Franklin and J. J. Pindborg, "The calcifying epithelial odontogenic tumor: a review and analysis of 113 cases," Oral Surgery, Oral Medicine, and Oral Pathology, vol. 42, no. 6, pp. 753-765, 1976.

[19] A. A. Owosho, B. Xu, A. Kadempour et al., "Metastatic solid tumors to the jaw and oral soft tissue: a retrospective clinical analysis of 44 patients from a single institution," Journal of Cranio-Maxillo-Facial Surgery, vol. 44, no. 8, pp. 1047-1053, 2016. 